\title{
Magnetic Shielding for the Fermilab Vertical Cavity Test Facility
}

\author{
Camille M. Ginsburg, Clark Reid, and Dmitri A. Sergatskov
}

\begin{abstract}
A superconducting RF cavity has to be shielded from magnetic fields present during cool down below the critical temperature to avoid freezing in the magnetic flux at localized impurities, thereby degrading the cavity intrinsic quality factor $Q_{0}$. The magnetic shielding designed for the Fermilab vertical cavity test facility (VCTF), a facility for CW RF vertical testing of bare ILC 1.3 GHz 9-cell SRF cavities, was recently completed. For the magnetic shielding design, we used two cylindrical layers: a room temperature "outer" shield of Amumetal (80\% Ni alloy), and a $2 \mathrm{~K}$ "inner" shield of Cryoperm 10. The magnetic and mechanical design of the magnetic shielding and measurement of the remanent magnetic field inside the shielding are described.
\end{abstract}

Index Terms - Magnetic shielding, Superconducting cavity resonators

\section{INTRODUCTION}

$\mathrm{T}$ HE Fermilab vertical cavity test facility (VCTF), for CW $\mathrm{RF}$ testing of $1.3 \mathrm{GHz}$ 9-cell superconducting niobium $\mathrm{RF}$ cavities for the International Linear Collider (ILC), was completed in July 2007. The primary purpose of the test facility is to assess the performance of cavities, both as a study of their production and processing, and as an acceptance test prior to insertion in a cryomodule. The complete facility description is given in [1]. These cavities have to be shielded from magnetic fields present during cool down below the critical temperature to avoid freezing in the magnetic flux at localized impurities, thereby degrading the cavity intrinsic quality factor $\mathrm{Q}_{0}$.

A perfect type-II superconductor such as niobium will expel all magnetic flux below the critical temperature, for magnetic fields smaller than the lower critical field, $\mathrm{H}_{\mathrm{cl}}$. However, for the high-quality niobium used for SRF cavity construction, the magnetic flux has been experimentally found to be fully trapped when cavities are cooled in ambient magnetic fields smaller than 3 Oe [2].

Manuscript received 19 August 2008. The work herein has been performed at Fermilab, which is operated by Fermi Research Alliance, LLC under Contract No. DE-AC02-07CH11359 with the United States Department of Energy.

Camille M. Ginsburg, Clark Reid, and Dmitri A. Sergatskov are with Fermilab, Batavia, IL 60510 USA (phone: 630-840-3901; fax: 630-840-2383; e-mail: ginsburg@fnal.gov).
The residual resistance which results from a cavity being cooled in a small remanent field is associated with a degraded cavity intrinsic quality factor $\mathrm{Q}_{0}$ via

$$
Q_{0}=\frac{G}{R_{s}}
$$

where $\mathrm{G}$ is the cavity geometric factor and $R_{s}$ is the residual surface resistance given by

$$
R_{s}=R_{B C S}+R_{r e s}+R_{\text {mag }} .
$$

$R_{B C S}$ is the BCS resistance and $R_{\text {res }}$ is the residual surface resistance due to the condition of the cavity surface. $R_{\text {mag }}$ is the resistance due to trapped magnetic flux and for a niobium SCRF cavity is

$$
R_{\text {mag }}=\eta\left[\frac{n \Omega}{m O e}\right] H_{0}[m O e] n \Omega
$$

where $H_{0}$ is the external magnetic field, and the factor $\eta$ varies experimentally from about $0.4 \mathrm{n} \Omega / \mathrm{mOe}$ for RRR values of $\sim 180$, up to $1.4 \mathrm{n} \Omega / \mathrm{mOe}$ for $\mathrm{RRR}$ values of $\sim 30$, as shown in a compilation in Ref. [3]. Furthermore, for the RRR range studied, 30 to 180 , the value of $\eta$ was found [3] to follow the relation

$$
\eta=1.9 \times 10^{-4} \sqrt{\frac{f[H z]}{R R R}} \frac{n \Omega}{m O e}
$$

where $f$ is the cavity resonant frequency. For ILC cavities, the niobium should have RRR of at least 300 [4], which is rather higher than the range studied in [3]. The magnetic shielding was designed to attenuate the ambient field to $\lesssim 10 \mathrm{mOe}$ to keep $R_{\text {mag }}$ below $10 \mathrm{n} \Omega$ for ILC cavities at $2 \mathrm{~K}$, so that the irreducible $\mathrm{BCS}$ losses dominate the residual resistance.

Because the VCTF was built in a building which has housed many high-field magnets, there had been some concern that the rebar in the floor or other structural iron near the cryostat could have been magnetized. Therefore, before starting construction of the pit, the magnetic field in the vicinity was measured and verified to be consistent with the Earth's magnetic field [5].

\section{Electromagnetic Design of MAGnetic ShIELD}

The attenuation $(A)$ is defined as the magnetic field without the shield divided by the magnetic field with the shield. For a single, open-ended cylindrical shield with thickness $(t)$ much smaller than diameter $(D)$, and permeability $(\mu)$ much greater than one, the attenuation factors transverse $(\perp)$ and parallel $(\|)$ to the cylinder axis suitable for a static field are [6] 


$$
\begin{aligned}
& A_{\perp} \cong \frac{\mu t}{D}+1 \\
& A_{\|} \cong 4 N A_{\perp}+1
\end{aligned}
$$

where $\mathrm{N}$ is the demagnetizing coefficient of a prolate spheroid [7]

$$
N=\frac{1}{\left(\frac{L}{D}\right)^{2}-1}\left[\frac{\left(\frac{L}{D}\right)}{\sqrt{\left(\frac{L}{D}\right)^{2}-1}} \ln \left(\left(\frac{L}{D}\right)+\sqrt{\left(\frac{L}{D}\right)^{2}-1}\right)-1\right]
$$

For two open-ended cylinders, inner (1) and outer (2) [3]

$$
\begin{aligned}
& A_{\perp} \cong 1+\frac{\mu_{1} t_{1}}{D_{1}}+\frac{\mu_{2} t_{2}}{D_{1}}+\left(\frac{\mu_{1} t_{1}}{D_{1}}\right)\left(\frac{\mu_{2} t_{2}}{D_{2}}\right)\left[1-\frac{D_{1}^{2}}{D_{2}^{2}}\right] \\
& A_{\mid} \cong 1+4 N\left(\frac{\mu_{1} t_{1}}{D_{1}}+\frac{\mu_{2} t_{2}}{D_{2}}\right) \\
& +\left\{\begin{array}{l}
\left.\left[N\left(\frac{\mu_{1} t_{1}}{D_{1}}\right)\left(\frac{\mu_{2} t_{2}}{D_{2}}\right)\right]\left(\frac{D_{1}+D_{2}-t}{2 L_{1}+0.5\left(D_{1}+D_{2}-t\right)}\right)\right) \\
\times\left[1-\left(\frac{D_{1}}{D_{2}-t}\right)^{2}\right]
\end{array}\right\}
\end{aligned}
$$

$N$ is the outer shield demagnetizing coefficient. To ensure that the shield will not saturate in the ambient field, the field in the shield has to always be less than the saturation field for the material, i.e., $H_{s}=(D / t) H_{0}$.

Shielding manufacturers supply a magnetic permeability for shielding applications in a field of 40 Oe, $\mu_{40}$. Our assumption, based on experience at other laboratories, was that the permeability would be a factor 4-5 lower for the very low fields in this application, and for a realistically formed, treated and assembled shield.

For the geometry and ambient field of the VCTF cryostat, it was determined that a room temperature shield made of Amumetal [8], an $80 \% \mathrm{Ni}$ alloy with $\mu_{40} \sim 60000$ and $H_{s} \sim 8000$ Oe, and a $2 \mathrm{~K}$ Cryoperm 10 shield [9] with $\mu_{40} \sim 65000$ and $H_{s} \sim 9000$ Oe would shield the cavities to $\lesssim 10 \mathrm{mOe}$ without danger of saturation, leaving sufficient margin for the uncertainty on $\mu$.

\section{MechanicAl Assembly of Magnetic ShIELD}

The two-layer cylindrical magnetic shielding design incorporates a room temperature outer shield outside the vacuum vessel, and an inner shield inside the helium vessel. The outer shield is made of $1 \mathrm{~mm}$ thick Amumetal, with a length of $4216 \mathrm{~mm}$ and an inner diameter of $1070 \mathrm{~mm}$. It was built in half-cylinder pieces which were tightened against the vacuum vessel outer diameter using Amumetal joiner bands with PEM [10] fasteners and 304 stainless steel screws. Additional support is provided by turn buckles between the top of the shielding and the vacuum vessel top flange. The inner shield has one perforated end cap at the bottom (for LHe flow), and is made of $1 \mathrm{~mm}$ thick Cryoperm 10, with a length of $3378 \mathrm{~mm}$ and an inner diameter of $635 \mathrm{~mm}$. It was also built in half-cylinder segments, which are butt-welded together into three sections; the final assembly is made using Cryoperm10 joiner bands with a stitched fillet weld on lap joints. It is attached to the outer surface of an aluminum cylinder for support and protection from damage during cavity insertion into the cryostat, using 304 stainless steel screws into tapped holes. The screws do not protrude past the aluminum inner diameter. The Cryoperm 10 shield assembly has been permanently installed in the cryostat. The mechanical assembly of the two-layer shield is shown in Fig. 1, along with the range of the magnetic field measurement described in Sec. IV.

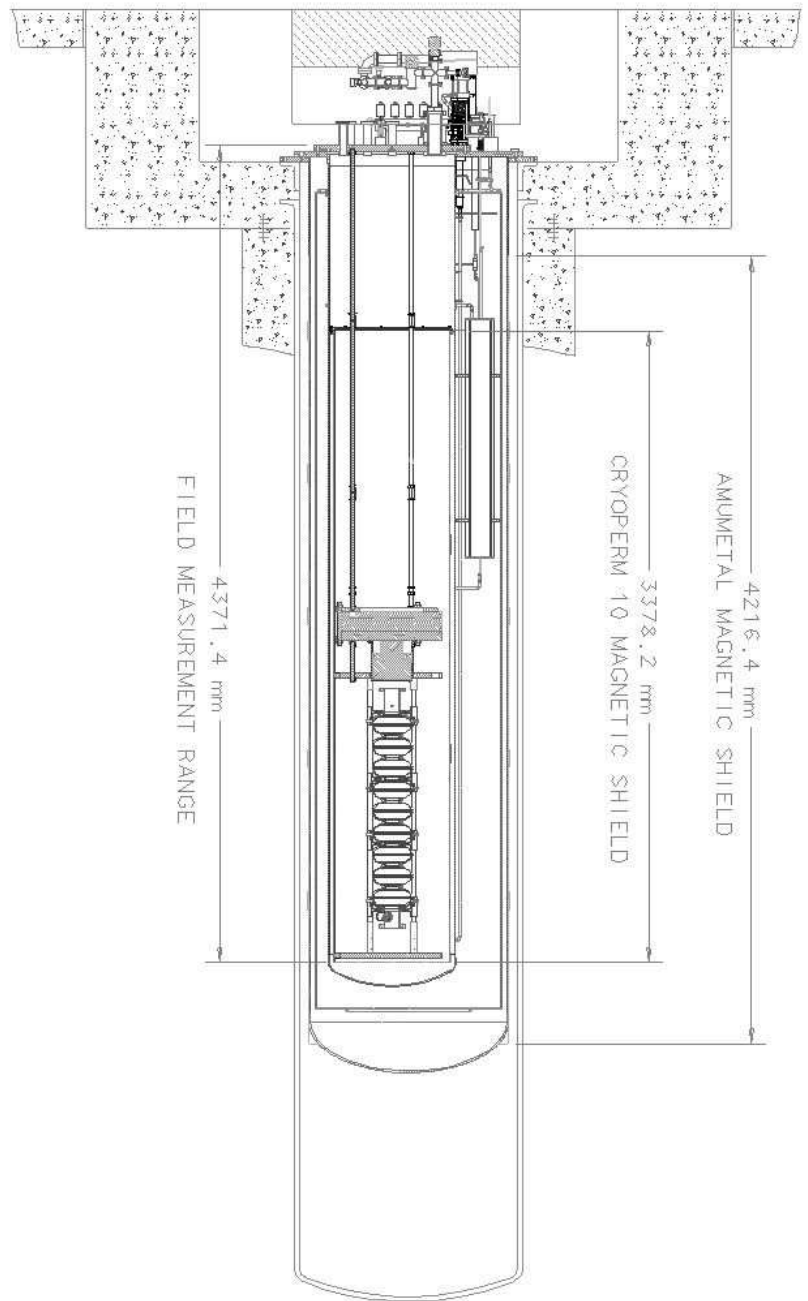

Fig. 1. The mechanical assembly of the magnetic shielding showing placement of the two-layer shielding at the VCTF cryostat and the field measurement range.

\section{Magnetic MeAsurements}

The magnetic field inside the cryostat has been measured as a function of depth, and at various azimuthal positions. The field was measured with a three-axis fluxgate sensor, Mag03MC1000 from GMW Associates [11], which is capable of measuring fields up to $10 \mathrm{Oe}$ with $0.5 \mathrm{mOe}$ offset accuracy and $0.01 \mathrm{mOe}$ resolution. The probe was attached to a power supply/controller unit Mag-03PSU, also from GMW, via a $5 \mathrm{~m}$ 
long cable. The controller was connected to a digital multimeter and read out via GPIB to a LabView program. The measurements were done at few fixed depths as the probe was lowered and raised inside the cryostat along a plastic guide tube. The tube could be positioned either in the center of the shield or in one of the six locations spread equidistantly near the perimeter of the shield. Multiple successive measurements were done at each physical location. The RMS of each set of measurements was less than $0.1 \mathrm{mOe}$. The measurements along the central axis were repeated at the beginning and at the end of the measurement sequence. The difference between these two series was of the order of 0.1 mOe in the field-flat region, near the center of the shield; the error increased to a few mOe in the high-field-gradient region near the shield edges. That suggests that the precision of the experiment was largely due to the positioning accuracy of the probe.

Three measurements were made: pit without magnetic shielding, pit with outer magnetic shield only, and pit with both the inner and outer magnetic shield. The magnetic field at the building floor in the pit region was measured to be consistent with the Earth's magnetic field $(\sim 0.5$ Oe), with some small local enhancements. The measurement with the cryostat and outer shield in place show that the field in the area of interest is less than $50 \mathrm{mOe}$, with a minimum of $\sim 40$ mOe. The permeability of the outer magnetic shield, as estimated from the minimum magnetic field, was determined to be about 40000 from (5) and (6). The measurement with both the inner and outer shield in place shows a minimum magnetic field of about 2 mOe. From (7) and (8), and assuming the permeability of Amumetal is 40000 from the previous measurement, the permeability of Cryoperm $10 \mathrm{at}$ room temperature is about 10000 . The permeability of the Cryoperm 10 is expected to increase significantly at $2 \mathrm{~K}$. Already at room temperature, the combined shielding has met the design goal of $\leqslant 10$ mOe. Fig. 2 shows the roomtemperature measurement of the magnetic field without shielding, with only the outer shielding, and with both the inner and outer shielding layers in place.

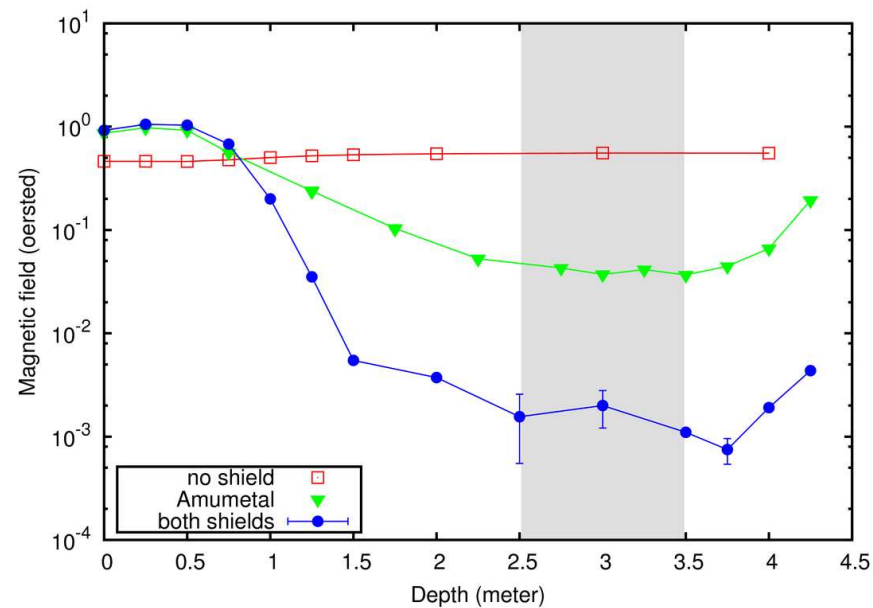

Fig. 2. Room temperature measurement of the magnetic field without shielding (squares), with only the outer Amumetal shielding (triangles) and with both the Amumetal and Cryoperm 10 shielding layers in place (circles). The cavity location is shown by the shaded region.

\section{MEASUREMENTS WITH A CAVITY}

Although a $2 \mathrm{~K}$ measurement of the magnetic field is desirable, it is challenging to put a room-temperature probe into a superfluid helium vessel. Instead, we extrapolate an upper limit for the ambient field at $2 \mathrm{~K}$ using cavity test data. Accel-6 is a nine-cell ILC-style cavity manufactured by Accel Corporation [12] using Wah Chang [13] niobium with a measured RRR of $\sim 500$. The cavity was not annealed before the test. The cavity $\mathrm{Q}_{0}$ was measured as a function of temperature from $4 \mathrm{~K}$ down to $1.42 \mathrm{~K}$. The surface resistance from (1), using $G=270 \Omega$ [14], is shown as a function of $1 / \mathrm{T}[\mathrm{K}]$ in Fig. 3. The data are fit to a function of the form

$$
R_{s}=\kappa \times \frac{1}{T} \times \exp \left(-\beta \frac{1}{T}\right)+R_{m a g}
$$

where $\kappa, \beta$, and $R_{\text {res }}$ are fit parameters. Only the data below $\mathrm{T}_{\lambda}$ $\left(1 / \mathrm{T}=0.46 \mathrm{~K}^{-1}\right)$ were used in the fit. The first term in (9) is $R_{B C S}$. We assume conservatively that $R_{\operatorname{mag}}$ is the sole contributor to the remaining surface resistance at $1.42 \mathrm{~K}$. The resulting residual resistance due to trapped magnetic flux is $R_{\text {mag }}=4.97 \pm 0.16 \mathrm{n} \Omega$.

From (3) and (4), for a RRR 500 ILC cavity, $\eta \sim 0.3$ and the upper limit on the magnetic field is $H_{0} \lesssim 17$ mOe.

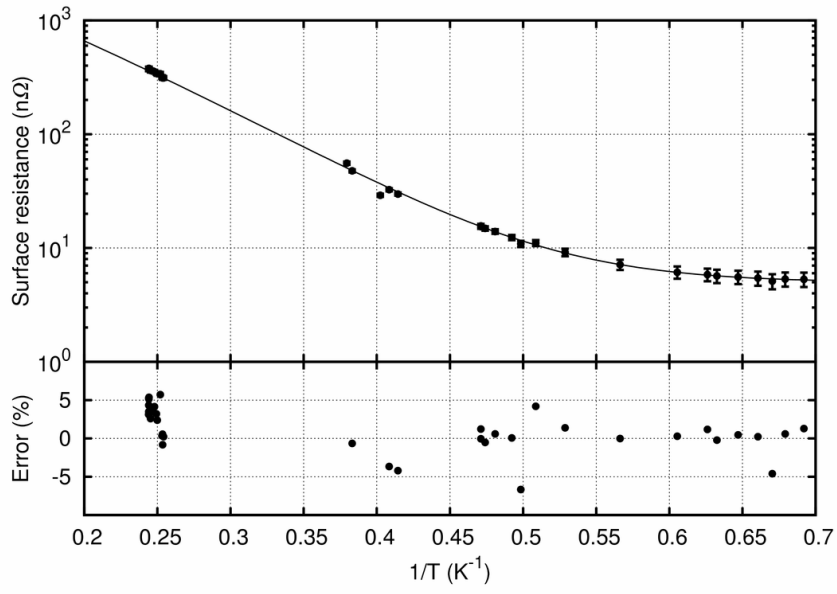

Fig. 3. The measurement of the residual resistance of the Accel- 6 cavity. Only the data below $\mathrm{T}_{\lambda}\left(1 / \mathrm{T}=0.46 \mathrm{~K}^{-1}\right)$ were used in the fit.

\section{CONCLUSION}

A two-layer magnetic shield has been designed and built for the Fermilab Vertical Cavity Test Facility. Measurements demonstrate that the design goal of attenuating the remanent field at the cavity to $\$ 10 \mathrm{mOe}$ has been reached. An extrapolation of the magnetic field at the cavity during a cavity test with the magnetic shielding in place is consistent with the measurement.

\section{ACKNOWLEDGMENT}

The authors are grateful to Cosmore Sylvester (Fermilab) for magnetic shielding procurement and engineering expertise, Mike Tartaglia (Fermilab) for magnetic measurements of ambient field in the VCTF area, Genfa Wu (Fermilab) for Accel-6 niobium data and related information, and Larry 
Maltin (Amuneal Manufacturing Corp.) for valuable discussions. The authors are indebted to the VCTF technical staff for their invaluable assistance in assembling and installing the magnetic shielding and for their superb support of the measurements described in this paper.

\section{REFERENCES}

[1] J. .P. Ozelis et al.,"Design and Commissioning of Fermilab's Vertical Test Stand for ILC SRF Cavities," WEPMN106, PAC'07, Albuquerque, NM, USA, June 2007.

[2] C. Vallet et al., "Flux trapping in superconducting cavities," Proceedings of EPAC92, Berlin, Germany, p.1295, March 1992.

[3] C. Vallet, "Etude de la Dissipation dans les Supraconducteurs en Regime Haute Frequence, ” Ph.D. Thesis, L’Universite Claude Bernard Lyon I, 1994.

[4] International Linear Collider Reference Design Report, 2007, http://ilcdoc.linearcollider.org/record/6321/files/ILC_RDR_Volume_3Accelerator.pdf.

[5] M. Tartaglia (Fermilab), private communication.

[6] Albrecht J. Mager, Magnetic Shields, IEEE Transactions on Magnetics 6, (1970) 67.

[7] R. Bozorth, Ferromagnetism, IEEE Press, New York, 1951, p.849.

[8] Amuneal Manufacturing Corp., Philadelphia, PA, http://www.amuneal.com/

[9] Vacuumschmelze GmbH, Hanau, Germany, http://www.vacuumschmelze.de/.

[10] Penn Engineering and Manufacturing Corp., Danboro, PA, http://www.pemnet.com/.

[11] GMW Associates, Inc., San Carlos, CA, http://www.gmw.com.

[12] Accel Instrument GmbH, Bergisch Gladbach, Germany, http://www.accel.de/.

[13] Wah Chang, Albany, OR, http://www.wahchang.com/.

[14] B. Aune et al., "The Superconducting TESLA Cavities," Phys. Rev. ST Accel. Beams 3, 092001 (2000). 